\title{
Hidden velocity ordering in dense suspensions of self-propelled disks
}

\author{
Lorenzo Caprini $\odot^{*}$ and Umberto Marini Bettolo Marconi \\ Dipartimento di Fisica, Universitá di Camerino, Via Madonna delle Carceri, I-62032 Camerino, Italy \\ Claudio Maggi, Matteo Paoluzzi $\odot$, and Andrea Puglisi \\ CNR-Istituto Sistemi Complessi, P. le A. Moro, I-00185 Rome, Italy
}

(Received 5 April 2020; accepted 18 May 2020; published 11 June 2020)

\begin{abstract}
Recent investigations of the phase diagram of spherical, purely repulsive, active particles established the existence of a transition from a liquidlike to a solidlike phase analogous to the one observed in colloidal systems at thermal equilibrium. In particular, an intermediate hexatic phase is observed in two dimensions. At variance with previous studies, we highlight the dynamical anomalies of dense active phases employing suitable parameters accounting for the observed spatial velocity correlations. The resulting information is encoded into a phase diagram evidencing the nonequilibrium features of self-propelled systems at a high density. First, we unveil the growth — with density and activity — of ordered domains where the particles' velocities align in parallel or vortexlike domains, extending the preliminary observation found in the phase-coexistence regime. Second, when activity is strong, the spatial distribution of the kinetic energy becomes heterogeneous, with high-energy regions correlated with defects of the crystalline structure. This spatial heterogeneity is accompanied by temporal intermittency, with sudden peaks in the time series of kinetic energy. The observed dynamical anomalies cannot be detected by considering only the structural properties of the system and are exquisitely nonequilibrium peculiarities absent in dense equilibrium colloids.
\end{abstract}

DOI: 10.1103/PhysRevResearch.2.023321

\section{INTRODUCTION}

The dynamics of colloidal particles at high densities have been widely explored, theoretically, numerically, and experimentally, in the last decades [1,2]. At thermodynamic equilibrium, the Mermin-Wagner theorem rules out the existence of a crystalline phase in two-dimensional systems, characterized by long-range translational order [3,4]. As shown by Halperin and Nelson [5] and Young [6], in two dimensions the melting transition proceeds via two continuous Berezinskii-KosterlitzThouless $[7,8]$ transitions driven by topological defects, i.e., a hexatic-liquid transition, with a quasi-long-range orientational order, and a solid-hexatic transition, characterized by quasi-long-range translational order and long-range orientational order [6,9]. Density and temperature are the control parameters to move from liquid to hexatic and to solidlike aggregation phases. This scenario has been verified employing dense suspensions of equilibrium colloids [10,11].

Recently, the study of two-dimensional systems of selfpropelled particles at high packing fractions has attracted the attention of the active matter community [12,13], since it may offer interesting engineering applications, for instance,

\footnotetext{
*lorenzo.caprini@gssi.it

Published by the American Physical Society under the terms of the Creative Commons Attribution 4.0 International license. Further distribution of this work must maintain attribution to the author(s) and the published article's title, journal citation, and DOI.
}

in the design of new materials [14]. Although most of the experimental studies have so far focused on the low-density regime, some novel experiments have investigated Janus particles also in the case of very dense suspensions [15]. Another interesting class of high-density nonequilibrium systems is represented by driven granular media where monodisperse polar grains under shaking [16] display persistent motion. It is also worth mentioning some recent studies of artificial microswimmers at such densities [17], revealing an intriguing experimental scenario for nonequilibrium aggregation. Specimens of active matter systems at very high densities are very interesting also in the biological realm. Typical examples are tissues composed of highly packed eukaryotic cells. Particular attention has been devoted to the dynamics of confluent monolayers [18,19], which slows down as the density increases. More recently, an amorphous "solidification" process has been investigated during the process of vertebrate body axis elongation [20], where cells become solidlike.

Theoretical approaches in the statistical physics of active matter focus on simplified models of self-propelled particles, the active Brownian particle (ABP) being one of the most studied. Despite the existence of a vast literature concerning the regime of moderate packing fractions, $\mathrm{ABP}$ dynamics in the high-density regime is by far less explored. For instance, active crystallization is studied in Ref. [21], where a shift of the liquid-solid transition line towards higher densities with respect to the Brownian counterpart is revealed. In addition, this transition is accompanied by a true nonequilibrium phenomenon: liquid and solid phases are separated by a region where the suspension is globally ordered but bubbles 
(a)

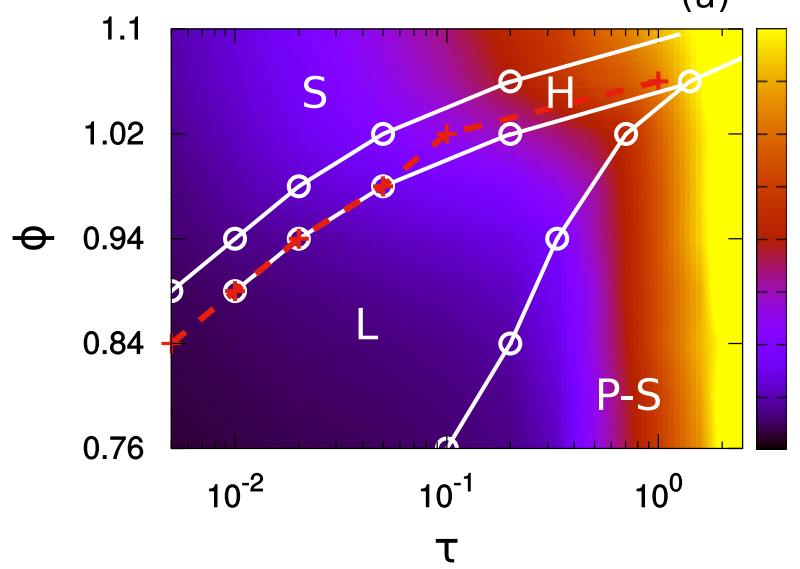

(b)

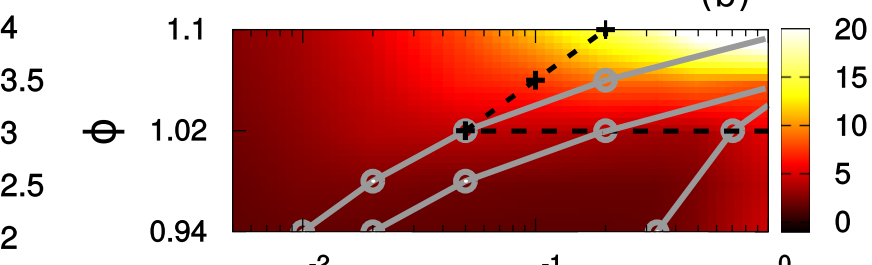

1.5

1

0.5

(c)

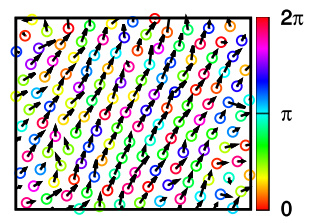

$10^{-1} \quad 10^{0}$

(d)

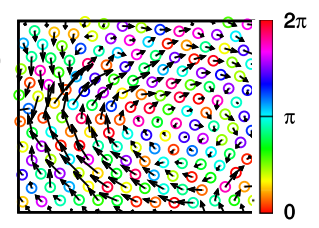

FIG. 1. Dynamical phase diagram. (a) Phase diagram as a function of $\tau$ and $\phi$, with each region's color depending on the size of the aligned domains, quantified by the parameter $R / \sigma$. Phases: $\mathrm{S}$, solid; $\mathrm{H}$, hexatic; L, liquid; and P-S, phase separated. Phases are separated by solid lines. The dashed red line was obtained by monitoring the pair correlation function, $g(r)$. (b) The top region of the phase diagram, with colors depending on the correlation length, $\ell$, of the spatial velocity correlation. The dashed black line delimits the regions where kinetic energies show an intermittent phenomenology. (c), (d) Two zooms of the snapshot configuration realized at $\phi=1.1$ and $\tau=1$. Colors encode the orientation of the self-propulsion, while black arrows represent the velocity vectors. (c) An aligned domain. (d) A vortexlike structure in the pattern of the particles' velocities. Simulations are obtained with $v_{0}=50$ and the interparticle interaction discussed in the text.

of "liquid" persist. A more detailed analysis revealed the occurrence of traveling crystals [22,23], accompanied by a transition to rhombic, square, and even lamellar patterns. This phenomenology has recently been confirmed by experiments realized with vibrating granular disks [24].

The construction of the phase diagram of the ABP model [25-27] follows the idea that its behavior [28] for small active forces resembles that of passive Brownian particles, with the occurrence of "gaslike," "liquidlike," "hexaticlike," and "solidlike" phases, with a shift of the transition lines towards higher densities when activity increases $[25,28]$. On the contrary, for large self-propulsion (but moderate densities) an unexpected phenomenon occurs: the system phase separates even in the absence of attractive interactions [26,29-36] (socalled motility-induced phase separation (MIPS) [37-40]). Differently, at high densities but far from equilibrium, "standard" crystallization seems to occur $[21,25]$. The general picture suggested by these studies is that the highdensity equilibrium scenario extends, qualitatively identically, to active systems, with the only difference being that selfpropulsion may induce a phase separation or destabilize the ordered phases. The systems being far from equilibrium, this conclusion is, somehow, counterintuitive. In the present paper, we show that it can even be wrong.

Several studies have already suggested that the MIPS region displays a richer picture with respect to the passive phases in the coexistence region. Analysis of the pressure $[41,42]$ and the interfacial tension between the gas and the cluster phase [43,44] provided the first clue supporting this statement. Recently, the study of the particles' velocities revealed unexpected features which are certainly absent in equilibrium fluids, e.g., different kinetic temperatures inside and outside the dense cluster [45] and spontaneous alignment of velocities in the phase-separated regime [46].

Given the relevance of dense active phases in both experimental and theoretical studies, the present paper is entirely devoted to understanding the nonequilibrium features of dense phases of self-propelled disks: we show that the peculiarity of active dense systems lies in their dynamical properties and, in particular, in their interplay with the structural properties. In this framework, the presence of translational and/or orientational orders plays a fundamental role.

\section{Summary of results}

Our main findings can be summarized by the introduction of dynamical information about the particles' velocities into the structural phase diagram, as shown in Fig. 1. We enrich the picture reported in $[25,28]$ which could lead to the misleading conclusion that the only relevant aspect concerning the behavior of active high-density systems is the shift of liquid-hexatic and hexatic-solid transitions. The phase diagram concerns the two-dimensional high-density regimes (both homogeneous and phase separated), with two main control parameters: the persistence time, $\tau$, of the active force (which is proportional to the Péclet number and inversely proportional to the rotational diffusivity) and the packing fraction, $\phi$. We recall the definition of $\phi=N \sigma^{2} \pi / 4 L^{2}, N / L^{2}$ being the number density and $\sigma$ the particles' diameter. Our "augmented" phase diagram challenges the widespread idea that the structural properties alone are enough to describe the dense phases of self-propelled particles and suggests that a richer picture is obtained by including velocity correlations, which, in turn, represent an exquisitely off-equilibrium feature of active systems.

Figure 1(a) portrays the phase diagram as a function of $\phi$ and $\tau$, which reproduces [25], with three homogeneous phases, i.e., the solid (S) phase, the hexatic $(\mathrm{H})$ phase, and the liquid (L) phase, and a nonhomogeneous regime with MIPS-like phase coexistence (P-S) (see Sec. II B for details). Our first finding is that the alignment of particles' velocities discovered in [46] in the P-S regime is observed also in 
the homogeneous liquid, hexatic, and solid phases. Particles' velocities form patterns, becoming arranged in aligned or vortexlike domains, as shown by the arrows in Figs. 1(c) and 1(d), even if the orientation of self-propulsion has negligible order (see the color coding in the same panels). The size of aligned domains-quantified by $R$, defined later, encoded by colors in Fig. 1(a)—increases as $\tau$ and $\phi$ increase, as discussed in detail in Sec. III A. The first new insight in this paper regards the analysis of the "nontrivial" interplay between structural properties and alignment domains. In the homogeneous liquid configurations, the size of the aligned velocity domains is rather small as a result of the absence of translational order, at variance with the solid (denser) case where the sizes reach large values. In the nonhomogeneous configurations, only an increase in $\tau$ induces an increase in the correlation length as a result of density saturation. We recall that order in the velocity field is absent in any passive Brownian suspension, even at high densities: it is, in fact, a pure nonequilibrium feature due to the presence of propulsion forces in the active dynamics. Interestingly, a similar effect is also observed in fluidized granular materials, where it is caused by the presence of dissipative interactions [47-49].

The second insight in this paper regards the occurrence of pattern formation in the particles' kinetic energies accompanied by temporal intermittent behaviors with regions of high velocity. These anomalies are caused by the lack of orientational order, confirmed by the correlation between the kinetic energy field and structural defects in the crystal arrangement (see details in Sec. III B). This phenomenology represents the main qualitative difference between active solid and hexatic phases. The results of this analysis are gathered in Fig. 1(b), which focuses on the top part (highest densities) of the phase diagram: the colors encode different information here, i.e., the correlation length, $\ell$, of the spatial velocity correlation, which takes into account also the kinetic energy (square modulus of the velocity) and not just the orientation of the velocity vectors as in the case of $R$ in Fig. 1(a). We observe that $\ell$ increases in the solid phase and saturates in the hexatic or liquid phases. This is mainly caused by the absence of translational and/or orientational order (it increases again in the phase-separated regime as explained in detail in Sec. III C). In the same panel, the dashed black line delimits a region where heterogeneous spatial distributions and temporal intermittent behaviors of the kinetic energy are observed.

The article is structured as follows: in Sec. II, we introduce the ABP model for interacting self-propelled particles, summarizing the structural properties of the system. In Sec. III, we present a detailed study of all the dynamical anomalies in the velocity orientation, velocity vector, and kinetic energy fields, correlating these anomalies with the different structural properties of the system. A theoretical approach is also presented in Sec. III C that allows us to predict the features of the spatial correlation functions of the velocity field. Section IV is devoted to conclusions and perspectives.

\section{THE SYSTEM OF INTERACTING SELF-PROPELLED PARTICLES}

We study a system of $N$ interacting ABP disks in two dimensions moving in a fluid at high viscosity (low Reynolds number). We neglect both hydrodynamic interactions among the particles and inertial terms [14]. The center of mass of each disk, $\mathbf{x}_{i}$, evolves according to the stochastic differential equation

$$
\gamma \dot{\mathbf{x}}_{i}=\mathbf{F}_{i}+\mathbf{f}_{i}^{a},
$$

where $\gamma$ is the drag coefficient of the fluid and the effect of the thermal noise due to the solvent is assumed to be much smaller than the effect due to the random active force $\mathbf{f}_{i}^{a}$ [14]. The term $\mathbf{F}_{i}$ represents the force contribution due to steric interactions, such that $\mathbf{F}_{i}=-\nabla_{i} U_{\text {tot }}$, where $U_{\text {tot }}=$ $\sum_{i<j} U\left(\left|\mathbf{x}_{i j}\right|\right)$, with $\mathbf{x}_{i j}=\mathbf{x}_{i}-\mathbf{x}_{j}$.

Following several studies in the literature [29,46], we choose $U(r)$ as a truncated and shifted Lennard-Jones potential:

$$
U(r)=\left\{\begin{array}{cl}
4 \epsilon\left[\left(\frac{\sigma}{r}\right)^{12}-\left(\frac{\sigma}{r}\right)^{6}\right]+\epsilon, & r \leqslant 2^{1 / 6} \sigma, \\
0 & r \geqslant 2^{1 / 6} \sigma .
\end{array}\right.
$$

The constant $\sigma$ represents the nominal particle diameter, while $\epsilon$ is the typical energy scale of the interaction. For numerical convenience, both these parameters are set to 1 in the simulations. Even in very packed configurations, each particle can only interact with its first neighbors due to the truncated potential.

The self-propulsion force, $\mathbf{f}_{i}^{a}=\gamma v_{0} \mathbf{n}_{i}$, evolves with ABP dynamics: $v_{0}$ is the modulus of the speed induced by the self-propulsion for a force-free particle, and $\mathbf{n}_{i}$ is a unit vector of components $\left(\cos \theta_{i}, \sin \theta_{i}\right)$. The orientational angle, $\theta_{i}$, performs angular diffusive motion described by

$$
\dot{\theta}_{i}=\sqrt{2 D_{r}} \xi_{i},
$$

where $\xi_{i}$ is a white noise with unit variance and zero average. The constant $D_{r}$ represents the rotational diffusion coefficient and its inverse defines the typical relaxation time, $\tau=1 / D_{r}$, of the active force [50].

We remark that no explicit aligning force is included in the present model, at variance with Vicsek-like models where particles' velocities are forced to align with the mean orientation of surrounding particles' velocities [51-53]. Thus, in contrast with Vicsek-like models, the dynamics, (1), does not produce any polarization of the directors $\mathbf{n}_{i}$. We also avoid employing any form of self-alignment between the particle velocity and the self-propulsion force responsible for orientation-velocity ordering as recently proposed in [54,55].

\section{A. Effective velocity dynamics of the particle}

In order to obtain theoretical predictions and interpret the results, following [46], it is useful to switch from the set of variables $\left\{\mathbf{x}_{i}, \mathbf{f}_{i}^{a}\right\}$ to the transformed variables $\left\{\mathbf{x}_{i}, \mathbf{v}_{i}\right\}$, eliminating the self-propulsions in favor of the particles' velocities, $\mathbf{v}_{i}=\dot{\mathbf{x}}_{i}$. We emphasize that the vectors $\mathbf{f}_{i}^{a}$ and $\mathbf{v}_{i}$ are not aligned, because of the interaction force $\mathbf{F}_{i}$. This is true, in particular, at high densities. The transformed dynamics reads

$$
\begin{gathered}
\dot{\mathbf{x}}_{i}=\mathbf{v}_{i}, \\
\tau \gamma \dot{\mathbf{v}}_{i}=-\gamma \sum_{j=1}^{N} \boldsymbol{\Gamma}_{i j}\left(\mathbf{r}_{i j}\right) \mathbf{v}_{j}+\mathbf{F}_{i}+\tau \gamma \mathbf{k}_{i},
\end{gathered}
$$


where both $\mathbf{v}_{i}$ and $\mathbf{x}_{i}$ belong to the plane $x y$ and $\mathbf{r}_{i j}=\mathbf{x}_{i}-\mathbf{x}_{j}$. Each term $\boldsymbol{\Gamma}_{i j}$ is a $2 \times 2$ matrix whose elements are

$$
\Gamma_{i j}^{\alpha \beta}\left(\mathbf{r}_{i j}\right)=\delta_{i j} \delta_{\alpha \beta}+\frac{\tau}{\gamma} \nabla_{i \alpha} \nabla_{j \beta} U\left(\left|\mathbf{r}_{i j}\right|\right),
$$

where Latin indices identifying the particles assume the values $i, j=1, N$, while Greek indices stand for the spatial components $(x, y)$. The last addend in Eq. (5), $\mathbf{k}_{i}$, is a noise term which reads

$$
\mathbf{k}_{i}=v_{0} \sqrt{2 / \tau} \boldsymbol{\xi}_{i} \times \mathbf{n}_{i}=v_{0} \sqrt{2 / \tau} \boldsymbol{\xi}_{i} \times \frac{\gamma \mathbf{v}_{i}-\mathbf{F}_{i}}{\gamma v_{0}} .
$$

$\boldsymbol{\xi}_{i}$ is the stochastic vector with components $\left(0,0, \xi_{i}\right)$ normal to the plane of motion. At variance with the dynamics of $\mathbf{f}_{i}^{a}$, the modulus of $\mathbf{v}_{i}$ is not constant because of the term $\propto \boldsymbol{\xi}_{i} \times$ $\mathbf{F}_{i}$. The result can be easily generalized to the case of finite thermal noise, as shown in Appendix A, using the strategy from [56].

The deterministic part of Eq. (5) represents the dynamics of an underdamped passive particle under the action of spacedependent friction. Indeed, the first term on the right-hand side of Eq. (5) can be split into a contribution, $-\gamma \Gamma_{i i} \mathbf{v}_{i}$, representing a generalized Stokes force acting on the $i$ th particle, plus a second contribution, $-\gamma \sum_{j \neq i}^{N} \Gamma_{i j}\left(\mathbf{r}_{i j}\right) \mathbf{v}_{j}$, which depends on the particles' relative positions and velocities and vanishes in a passive system.

The ABP equation, (5), for the velocity strongly resembles the analogous equation of another schematic model of selfpropelled particles, namely, the active Ornstein-Uhlenbeck model (AOUP) [57-66]. Such a connection has recently been established in some studies $[67,68]$ : the only difference between the ABP and the AOUP arises from the noise term $\mathbf{k}_{i}$. In the latter case, $\mathbf{k}_{i}$ is a simple white noise acting on the velocity as an effective thermal bath. On the other hand, in the ABP, $\mathbf{k}_{i}$ is perpendicular to $\mathbf{n}_{i}$, the orientation of the active force, and is a multiplicative noise since its amplitude depends on both $\mathbf{v}_{i}$ and $\mathbf{F}_{i}$ through the unit vector $\mathbf{n}_{i}$. Also, note that (since $\xi_{i}$ has unit variance and $\mathbf{n}$ is a unit vector) the variances of the $\mathrm{ABP}$ and AOUP noises coincide, being $v_{0}^{2} / \tau$. We emphasize that, upon fixing $v_{0}$, the noise variance decreases in the large persistence regime.

\section{B. Known results and positional order}

In the present section, we illustrate the phase diagram for the values of the control parameters $\phi$ and $\tau$ (at fixed $v_{0}$ ) explored in this work. Our results are obtained through numerical solutions of Eqs. (1) in a square domain of size $L$ under periodic boundary conditions. In the considered interval of packing fractions $(\phi \in[0.78,1.1])$, a suspension of passive Brownian particles exhibits liquid, hexatic, and solid phases depending on the temperature. Our ABP phase diagram, realized under stationary conditions, is consistent with the findings of Di Gregorio et al. [25]. As shown by these authors, as $\tau$ is increased the liquid-hexatic and the hexatic-solid boundaries shift to larger values of $\phi$ and the hexatic region of the phase diagram is enlarged with respect to the passive equilibrium picture. In the whole phase diagram, a further $\tau$ increase determines the transition from homogeneous to inhomogeneous configurations (MIPS). We ascertain that our simulations reach a stationary regime in both cases and, in particular, when phase separation occurs. Since the dense cluster reaches average sizes, we conclude that there is no evidence of coarsening dynamics.

When the persistence is smaller than the time scale associated with the potential, i.e., when $\tau \ll(\nabla \cdot \mathbf{F}(\bar{x}) / \gamma)^{-1}$ $(\bar{x}$ being the average distance between neighboring particles, which is fixed by the density in any homogeneous configurations), we expect the same behavior as in passive Brownian particles [69,70]: particles are homogeneously distributed in the box and arranged in the solid, hexatic, or liquid phase, as shown in [25], depending on the interplay between $\phi$ and $\tau$. In this regime, the active force acts as a thermal bath with effective diffusivity $\sim v_{0}^{2} \tau$. Thus, the growth of $\tau$, at fixed $v_{0}$, can be mapped in the increase in the effective diffusivity in the corresponding Brownian system. Depending on the interplay between $\phi$ and $v_{0}^{2} \tau$, the system explores liquid, hexatic, or solid phases. The structural properties are detected by monitoring the behavior of the orientational order parameter [26,71], $\psi_{6}\left(\mathbf{x}_{i}\right)$, defined as $\psi_{6}\left(\mathbf{x}_{i}\right)=\sum_{j} e^{6 i \alpha_{i j}} / N_{i}$, where $\alpha_{i j}$ is the angle-with respect to the $x$ axis-of the segment joining the $i$ th and the $j$ th particle and the sum is restricted to the first neighbors of particle $i$, namely, $N_{i}$. In particular, we focus on the correlation function, $g_{6}\left(r=\mid \mathbf{x}_{i}\right.$ $\left.\mathbf{x}_{j} \mid\right)=\left\langle\psi_{6}\left(\mathbf{x}_{i}\right) \psi_{6}^{*}\left(\mathbf{x}_{j}\right)\right\rangle /\left\langle\psi_{6}^{2}\left(\mathbf{x}_{j}\right)\right\rangle$, to distinguish between different phases. While $g_{6}(r)$ is roughly constant with the distance in the solid phase, it decays as an inverse power law in the hexatic phase. Differently, in the liquid phase, $g_{6}(r)$ shows an exponential decay. Examples of the different decays of $g_{6}(r)$ in the three structural phases are shown in Fig. 2(a).

In addition, we also measure the pair correlation function, $g(r)$. At a high density, the arrangement of particles is close to the hexagonal lattice, so that the peaks of the $g(r)$ are placed at positions $\bar{x}, \sqrt{3} \bar{x}, 2 \bar{x}, \sqrt{7} \bar{x}, \ldots$, where $\bar{x}$ is the typical distance between neighboring particles, fixed by the density in any homogeneous configurations. It is noteworthy that $\bar{x}$ is quite smaller than $\sigma$, meaning that particles climb on the interacting potential due to the high densities. Each particle interacts only with its six neighbors due to the truncated nature of $U(r)$. In analogy with an equilibrium system, one can roughly identify the liquid phase with the parameter region where the second peak of $g(r)$ is not split. We see that for all values of the packing fraction, the peaks of the $g(r)$ decrease as $\tau$ is increased, in agreement with the interpretation of the self-propulsion in terms of effective diffusivity: fluidization occurs. The $g(r)$ is shown in Figs. 2(g) and 2(h) for several values of $\tau$ and two densities, $\phi=0.94$ and 0.82 , respectively: at a high density [Fig. $2(\mathrm{~g})]$, the system maintains a split second peak, while at a moderate density [Fig. 2(h)], the system shows a transition-increasing $\tau$-towards a single second peak where a liquidlike structure occurs [69]. Upon increasing $\tau$ beyond some threshold value (that depends on $\phi)$, represented by the dotted red line in Fig. 2(g), the curves representing $g(r)$ saturate, meaning that the internal structure is no longer influenced by the value of $\tau$. On the other hand, the shape of $g(r)$ changes towards less fluid configurations where the system shows phase separation or inhomogeneities. This is not a surprise since, in these cases, particles in the clusters attain a more compact configuration. 
(a)
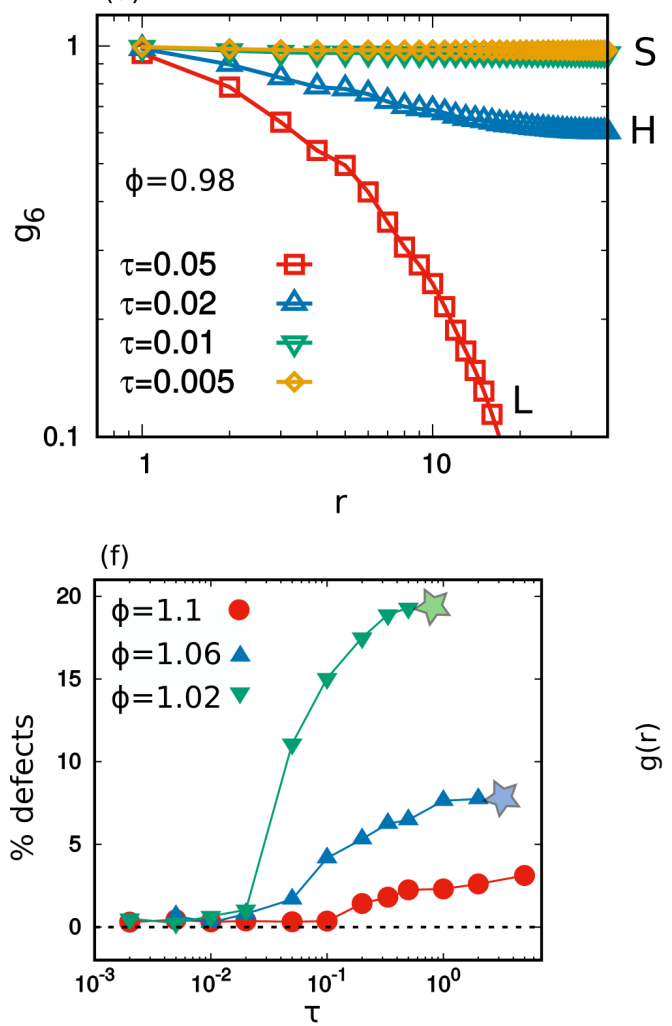

(b)

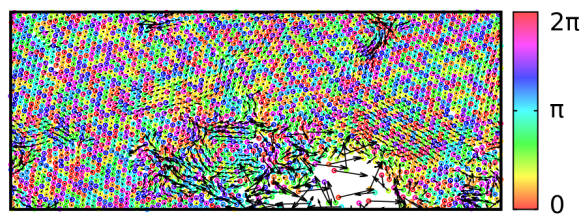

(c)

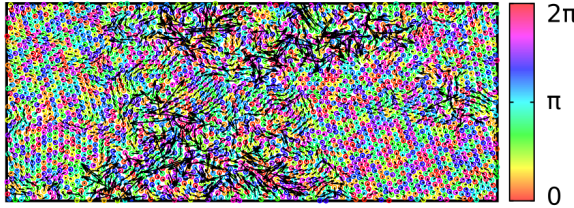

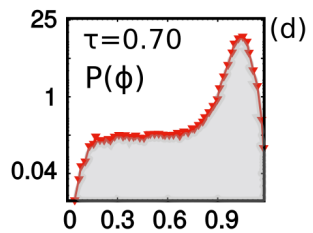

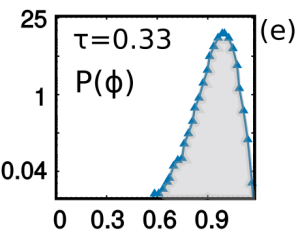

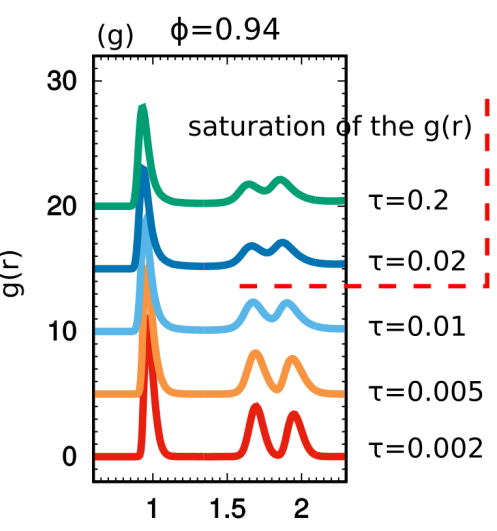

(h) $\phi=0.82$

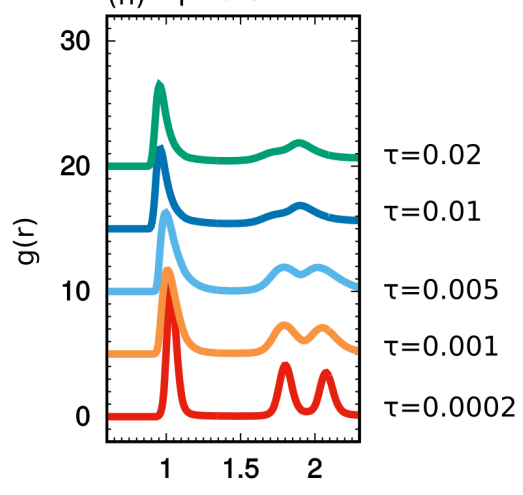

FIG. 2. Structural properties. (a) $g_{6}(r)$ at $\phi=0.98$ for different values of $\tau$, as reported in the legend, exploring the different structural phases: S, almost solid; H, hexatic; and L, liquid. (b), (c) Two snapshot configurations, where colors denote the orientation of the particles, namely, $\theta$. (d), (e) Distribution of the packing fraction, $P(\hat{\phi})$, corresponding to the two configurations. (f) The fraction of defects in the whole box vs $\tau$ for three values of the densities as illustrated in the legend. The colored stars show the value of $\tau$ at which the system becomes nonhomogeneous. (g), (h) A set of $g(r)$ obtained at different values of $\tau$ for $\phi=0.94$ and 0.82 , respectively. Each curve is shifted along $y$ for presentation reasons. Simulations were always realized with $v_{0}=50$ and the interaction discussed in the text.

The boundary line between the homogeneous and the inhomogeneous (phase-separated) regimes is obtained by monitoring the stationary distribution of the local packing fraction, $P(\hat{\phi})$, shown in Figs. 2(d) and 2(e) for two different configurations at the same densities and different $\tau$ 's. When the system is spatially homogeneous $P(\hat{\phi})$ displays small tails and a peak located at $\hat{\phi}=\phi$, while in the inhomogeneous region it displays a long tail for small $\hat{\phi}<\phi$ and a shift of the main peak for $\hat{\phi}>\phi$. The line of the transition from homogeneous $(\mathrm{L}, \mathrm{H}, \mathrm{S})$ to phase-separated $(\mathrm{P}-\mathrm{H})$ phases is tracked in Fig. 1(a), in correspondence with the first point showing such a shift.

Finally, the fraction of defects vs $\tau$ is measured for the denser configurations spanning solid and hexatic phases, as illustrated in Fig. 2(f). A defect is detected by counting the number of neighbors of a particle inside a circular radius of size $\sigma$ : if the number of neighbors is different from six, we mark this point as a defect. The measure is stopped when the system becomes inhomogeneous, in the proximity of the colored stars. We observe that the solid-hexatic transition takes place where the fraction of defects reaches $\sim 5 \%$.

\section{ORDER IN THE VELOCITIES}

Figures 1(c) and 1(d) are snapshots of the system representing the particles' positions and velocities for a large value of the persistence: they do not show MIPS, since at $\phi=1.1$ the density remains homogeneous in the considered range of $\tau$. In the case of interacting systems, the velocities of the particles, $\mathbf{v}_{i}$, represented by black arrows in Figs. 1(c) and 1(d), differ from $\mathbf{f}_{i}^{a}$ and, despite the absence of any alignment interactions, align and self-organize in large oriented domains. On the contrary, the self-propulsion $\mathbf{f}_{i}^{a}$ remains randomly oriented. The alignment of velocity orientations corresponds to the collective movements of large domains of particles. Such domains rearrange continuously with time and, sometimes, collapse into vortex structures, at variance with the well-known traveling bands occurring in the Vicsek-like models [52,72-74]. This phenomenology, occurring in the steady state, resembles qualitatively the scenario presented in [46] in the dense cluster of a configuration showing MIPS. Moreover, we discover that this nonequilibrium phenomenology is accompanied by the occurrence of large regions having the same kinetic energy and fast regions in the proximity of defects which induce intermittent behavior in the time trajectory of the kinetic energy (see Sec. III B).

Hereafter, this velocity order is studied quantitatively in terms of spatial alignment velocity correlations and suitable order parameters, useful to estimate the size of the domains. We find that there are different aspects of the velocity ordering phenomenology-(i) order in the orientation of the velocity vectors and (ii) order in the full velocity vectors-accounting 
(a)
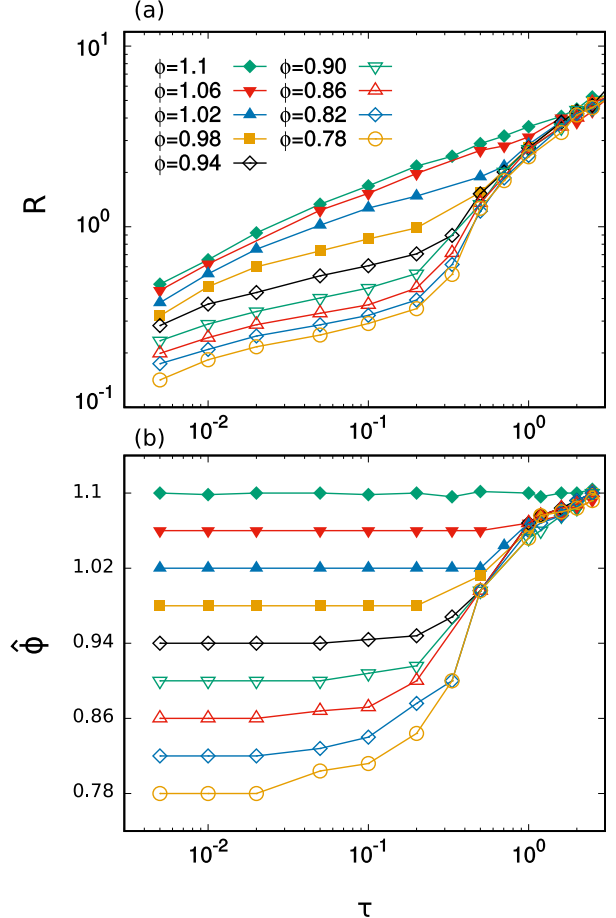

(c)

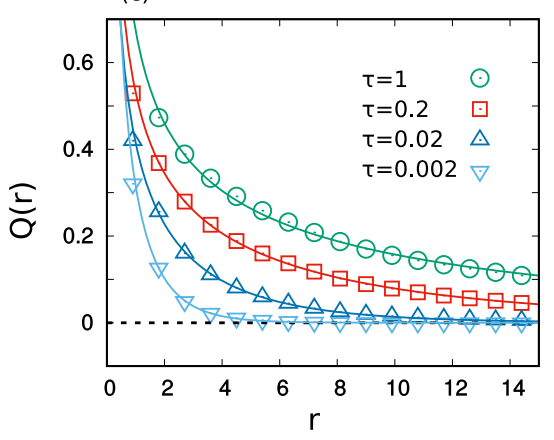

(e)

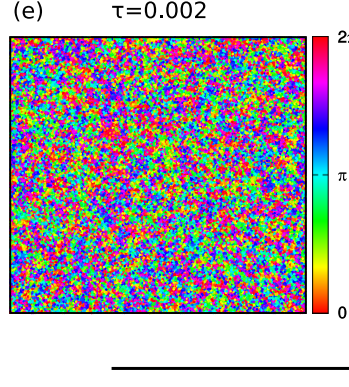

(f) $\quad \tau=0.05$ (d)
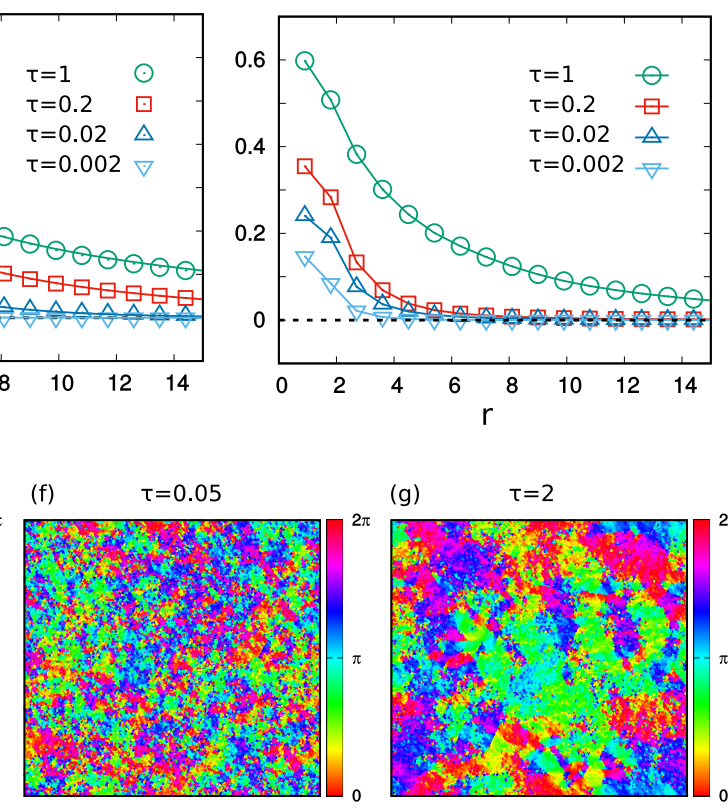

(g) $\quad \tau=2$

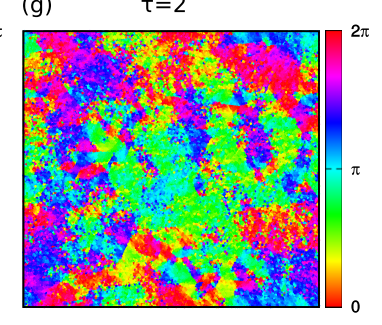

FIG. 3. Size of the velocity-aligned domains. (a), (b) $R$ and $\hat{\phi}$ as a function of $\tau$ for different values of $\phi$ as indicated in the legend. (c), (d) Spatial correlations of the velocity orientation, $Q(r)$, for $\phi=1.1$ and 0.94 , respectively. Curves with different $\tau$ values are reported according to the legend. (e), (f), (g) Three snapshot configurations, for $\tau=0.002,0.05$, and 2, respectively, with $\phi=1.1$. In these three panels, colors represent the orientation of the particles' velocities. All data in this figure were obtained at $v_{0}=50$.

also for the occurrence of large regions with the same kinetic energy.

\section{A. Velocity orientation}

For Vicsek-like models, the global alignment of the particles, also known as polarization, is commonly measured by the order parameters $[51,72,75,76]$

$$
\varphi=\frac{1}{N}\left|\sum_{i=1}^{N} e^{i \Theta_{k}}\right|,
$$

where $i$ is the imaginary unit and $\Theta_{k}$ is the velocity orientation of the $k$ th particle. This observable is almost 0 for particles without any alignment, typically at low numerical densities and high noise, and returns to 1 for perfectly aligned particles, e.g., for large values of $\phi$ [72]. On the contrary, in most systems of swimming active particles, typically evolved by means of overdamped equations, velocity vectors are ignored and the self-propulsion orientation is the only information used to characterize polarization: for instance, in the case of spherical (apolar) ABP particles, the parameter $\varphi$ in Eq. (8)with $\Theta_{k}$ replaced by $\theta_{k}$ from Eq. (3) - is close to 0 . In this model, since self-propulsions do not coincide with velocities, it is more suitable to consider the orientation of the velocity vector, $\dot{\mathbf{x}}_{k}$, in Eq. (8), i.e., replacing $\Theta_{k}$ with the angle formed by the velocity of the particle with respect to the $x$ axis. However, due to the presence of several domains with different orientations, there is no global velocity polarization. In principle, for very large persistence we could observe a large oriented domain spanning the whole box, but such a finite-size effect occurs only when $v_{0} \tau \gg L$, i.e., when the persistence length exceeds the size of the box [22,23]. We do not consider such a case in this paper.

A more appropriate indicator, which-even in the absence of a global polarization-provides information about the local alignment of the velocities and its dependence on physical parameters, is the spatial correlation function of the velocity orientation, $Q_{i}(r)$ [46], normalized to 1 . This observable measures the velocity alignment between particle $i$ and its neighboring particles located in the circular crown of thickness $\bar{r}=\sigma$ and mean radius $r=k \bar{r}, k$ being an integer positive number, and reads

$$
Q_{i}(r)=1-2 \sum_{j} \frac{d_{i j}}{\mathcal{N}_{k} \pi},
$$

where the sum runs over the particles within the circular crown defined by the value of $k$ and $\mathcal{N}_{k}$ corresponds to the number of particles contained in it. The term $d_{i j}$ is the angular distance between the two angles of the velocities of particles $i$ and $j$, namely, $\beta_{i}$ and $\beta_{j}$, calculated as $d_{i j}=$ $\min \left[\left|\beta_{i}-\beta_{j}\right|, 2 \pi-\left|\beta_{i}-\beta_{j}\right|\right]$. We average over all particles by defining $Q(r)=\sum_{i} Q_{i}(r) / N$, which (for $r>0$ ) has the property of being 1 and -1 for perfectly aligned and antialigned particles, respectively, and 0 in the absence of any form of alignment. In Figs. 3(c) and 3(d), we report $Q(r)$ for different values of the persistence time, $\tau$, and for two different densities. As expected, $Q(r)$ is a decreasing function of $r$. For the smallest values of $\tau$, the alignment is appreciable only in the first shells, a finding consistent with the scenario where the self-propulsion only acts as an effective thermal 
diffusion. Instead, as $\tau$ increases, $Q(r)$ takes on larger values in the first shell and decays more and more slowly towards 0 , with a typical decay length which roughly represents the average size of one domain: larger values of $\tau$ and density (almost always) produce both an increasing $Q(r)$ in the first shell $(k=1)$ and a slower decay of the whole function. This observation is, also, qualitatively confirmed by three snapshot configurations obtained for increasing values of $\tau$ from Fig. 3(e) to Figs. 3(f) and 3(g). There, the colors encode the velocity orientations, showing the increase in the average size of the individual velocity domains with $\tau$.

The velocity ordering is well captured by the parameter $R$, obtained by integrating over the whole box the correlation $Q(r)$ :

$$
R=\int Q(r) d r
$$

This parameter is a measure of the domain size and is studied in Fig. 3(a), as $\tau$ varies for several values of $\phi$, evaluating both the homogeneous and the nonhomogeneous configurations. We recall that the nonhomogeneous regimes correspond to the emergence of empty regions or phase separation, signaled by the presence of a nonsingle peak in the packing fraction distribution [see Figs. 2(d) and 2(e)]. To evaluate the impact of density inhomogeneity on $R$, we show the main peak position, $\hat{\phi}$ in Fig. 3(b), for different values of $\tau$ and average packing fraction $\phi$. We remark that $\hat{\phi} \approx \phi$ up to some critical value of $\tau$, then it increases. This critical value increases as the average packing $\phi$ is increased. At the larger packing fraction studied, $\phi=1.1$, the system remains homogeneous for all the explored values of $\tau$. It is noteworthy that the values of $\hat{\phi}$ (the densities in the denser part of the system) become independent of $\phi$ for $\tau$ sufficiently large.

As shown in Fig. 3(a), $R$ increases with $\tau$. For $\phi=1.1$, i.e., when the system is spatially homogeneous for all values of $\tau$, the growth is steady. This proves that the growth of $R$ occurs even in the absence of any local density inhomogeneity since it is not associated with some local change in density. Instead, for smaller values of $\phi$, a first slow monotonic increase is followed by a sharp one occurring at a value of $\tau$ for which the homogeneous liquidlike or hexatic phases break down in favor of an inhomogeneous phase. A comparison between Fig. 3(a) and Fig. 3(b) also suggests that $R$ has a strong dependence on $\phi$.

The occurrence of such a velocity order is evidence of the nonequilibrium nature of the dense active phases. Even if the structural (positional) information suggests an analogy with the liquid, hexatic, or solid phases of a passiveequilibrium-system, there is not an equilibrium counterpart of the velocity ordering phenomenon.

In general, it is believed that the occurrence of local velocity alignment is a consequence of the breaking of some microscopic isotropy (as occurs in the case of elongated particles [77-80]) or the introduction of explicit alignment interactions. The present ABP model subject to random independent active driving leads to velocity alignment — in the high-density regimes - even for spherical particles. This phenomenology simply arises from the interplay between self-propulsion and steric interparticle repulsion.

\section{B. Kinetic energy and intermittency}

Besides the local order of velocity orientations, spatial correlations also manifest in the speed, $v=|\mathbf{v}|$, and, thus, in the kinetic energy of the particles, $\propto v^{2}$. Figures 4(a)-4(c) show the map of $v^{2} /\left\langle v^{2}\right\rangle$ for three snapshot configurations obtained varying $\tau$, for $\phi=1.1$, i.e., at a density value such that the active system attains a solidlike state for every $\tau$. For small $\tau$, kinetic energies display uncorrelated spatial fluctuations (with Gaussian statistics; not shown here). As $\tau$ grows, structures characterized by similar (or correlated) values of $v^{2}$ appear, with alternation of fast and slow regions (each identified by a given color).

We also highlight an interesting connection between the kinetic energy spatial distribution and the structural properties of the system. In Figs. 4(d)-4(f), we plot the observable $\left|\psi_{6}\left(\mathbf{x}_{i}\right)\right|-$ which is the field pertaining to the crystalline orientational order-relative to the three configurations in Figs. 4(a)-4(c). The comparison between the maps of $\left|\psi_{6}\left(\mathbf{x}_{i}\right)\right|$ and $v^{2}$ reveals that the regions with high kinetic energies develop close to the defects of the crystalline structure. A similar scenario occurs for a smaller value of $\phi$, namely, $\phi=1.02$. In this case, the $v^{2}$ map is shown in Figs. 4(g)-4(i), while Figs. 4(j)-4(1) report the $\left|\psi_{6}\right|$ map. For this choice of $\phi$, the three values of $\tau$ distinguish different aggregation phases: phase separated, hexatic, and solid (from left to right). The solid phase for the smaller value of $\tau$ is qualitatively indistinguishable from the denser case [compare Figs. 4(c) and 4(i)]. Instead, for the intermediate value of $\tau$ [Fig. 4(h)] the occurrence of the hexatic phase is responsible for a larger number of defects and, thus, a larger number of mobile particles, as clearly shown from the comparison between Fig. 4(h) and Fig. 4(k). Finally, in the phase-separated configuration, the fastest regions are mostly concentrated near the boundary of the empty region [Fig. 4(g)].

To assume another perspective, it is instructive to consider the time behavior of the kinetic energy, $v^{2}$, calculated averaging over a box of size $l$ such that $\bar{r} \ll L$. This observable, as a function of time, is reported for different values of $\tau$ in Figs. 4(m) and 4(o) for $\phi=1.1$ and 1.02, respectively. In these two cases, the scenario is similar: for the smaller values of $\tau$ the kinetic energy displays symmetric and rapidly uncorrelated fluctuations around the mean value, $\left\langle v^{2}\right\rangle$. This is coherent with an effective equilibrium picture which is expected when $\tau \rightarrow 0$. For large values of $\tau$, sparse anomalous peaks manifest, corresponding to rare fluctuations, which move away from their average by several standard deviations. These peaks become higher and more isolated when $\tau$ increases. The observed behavior resembles the temporal intermittency observed in turbulence [81] or in active attractive glassy systems [82]. A more detailed analysis of this issue will be presented in a future work, while in this paper, we consider only the essential features of this phenomenon, in particular, the role played by defects in the solid phase: particles near the defects attain, in fact, a high kinetic energy. To corroborate our observation, we compare the fluctuations of the orientational order parameter, $\Psi_{6}=\sum_{i=1}^{N_{l}} \psi_{6, j} / N_{l}$, and those of the kinetic energy, $v^{2}$, both averaged over a box of size $l$ with $N_{l}$ particles. In particular, Fig. 4(n) shows the time trajectories of $1-\Psi_{6}$ and $v^{2}$, revealing a fair correlation between the occurrence of spikes for both these observables. 

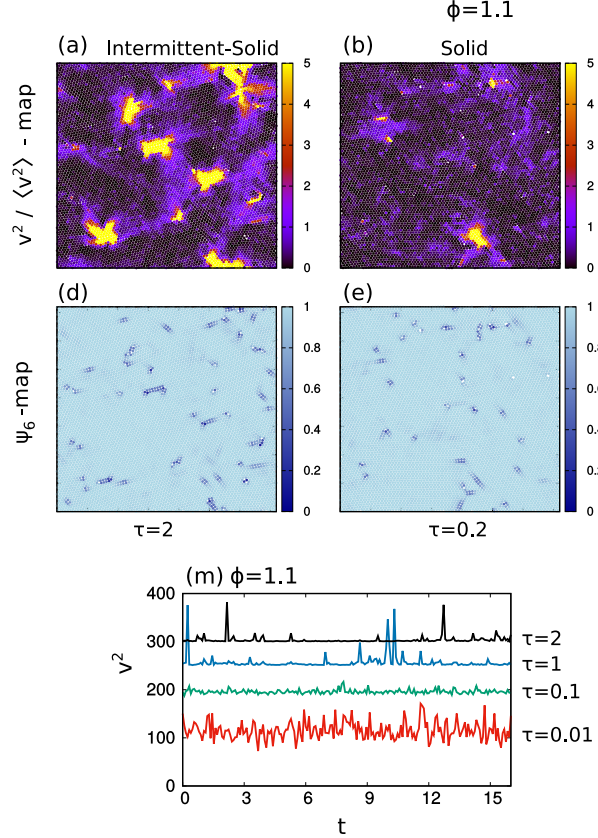
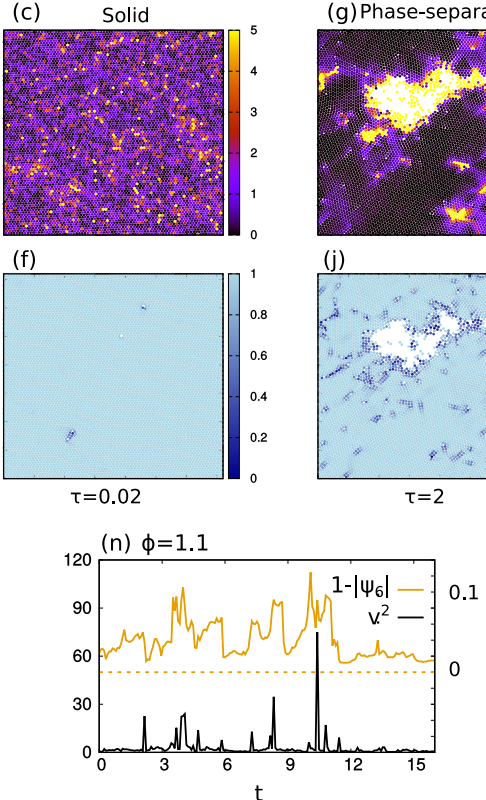

$\phi=1.02$
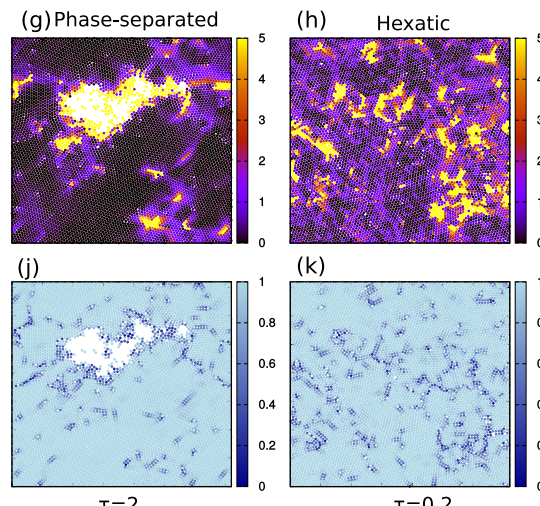

(k)
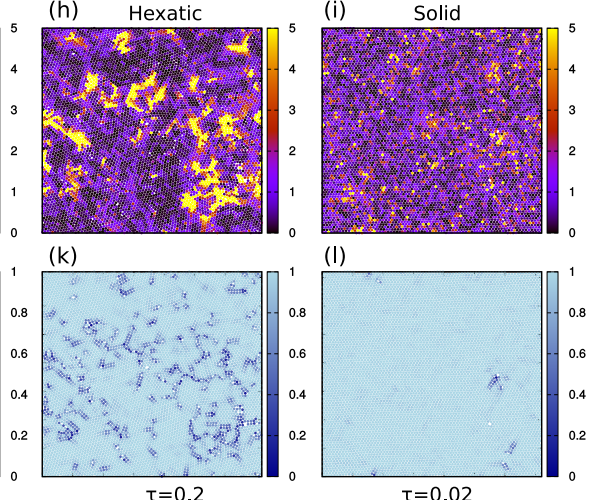

(I)
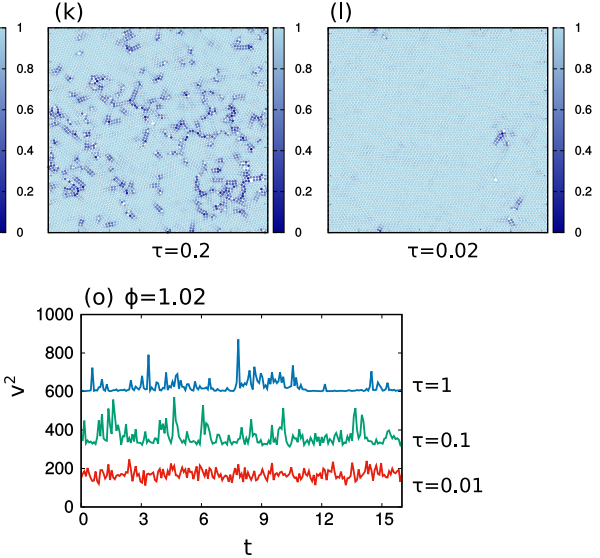

FIG. 4. Fluctuations of kinetic energy. Snapshots in the plane $x y$ realized at (a)-(f) $\phi=1.1$ and (g)-(l) $\phi=1.02$ for different values of $\tau$ as reported below the panels. Colors in (a)-(c) and (g)-(i) encode the value of the square velocity of each particle, $v^{2} /\left\langle v^{2}\right\rangle$, while colors in (d)-(f) and (j)-(l) represent the value of the crystalline orientational order parameter $\left|\Psi_{6}\right|$. (m, o) Different time series of $v^{2}$ obtained for $\tau=10^{-2}, 10^{-1}, 1$, and 2 as indicated in the legends, with $\phi=1.1(\mathrm{~m})$ and $\phi=1.02(\mathrm{o})$. (n) Comparison of a single trajectory of $v^{2}$ and $1-\left|\Psi_{6}\right|$, obtained with $\tau=2$ and $\phi=1$.1. All simulations were realized with $v_{0}=50$.

With the aim of introducing also the information on intermittency in the general picture, we have drawn a dashed black line in the phase diagram in Fig. 1: the line identifies the intermittency region and is tracked at the first values of $\tau$ and $\phi$ for which the peak of the $v^{2}$ trajectory overcomes 3 times the standard deviation from its average value.

\section{Vectorial velocity field}

In the previous sections, we have shown that the spatial correlation of velocity orientations increases with $\tau$, even in the presence of crystalline defects or large voids (such as those in the phase-separated regimes). Speed (velocity modulus) is more sensitive to the presence of defects and creates patterns with sparse strong fluctuations. A natural question arises: What happens to the spatial correlation of the full velocity vectors (which incorporate both orientation and modulus)?

A quantitative measure of the ordering of the velocities can be obtained by measuring the spatial correlation function in the steady state,

$$
C(r)=\frac{\langle\mathbf{v}(r) \cdot \mathbf{v}(0)\rangle}{\left\langle v^{2}\right\rangle},
$$

in the continuous limit, $\mathbf{v}_{i} \rightarrow \mathbf{v}(\mathbf{r}) \cdot\left\langle v^{2}\right\rangle$ is the variance of the velocity distribution calculated over the whole box. Our analysis limited to the case of homogeneous density under some assumptions is able to predict the form of $C(r)$ (as illustrated in Appendix B). The equation of motion, (5), is approximated by the AOUP dynamics, replacing the multiplicative noise with a two-dimensional additive noise. By this method, it is possible to predict the spatial velocity correlation function. At variance with the calculation reported in [46], here we assume that particles are free to oscillate around their equilibrium positions, i.e., they form a hexagonal crystal structure with oscillating sites. Under these simple hypotheses, an expression for $C(r)$ can be derived using the equation of evolution of the velocities. In particular, $C(r)$ displays an exponential-like behavior,

$$
C(r) \propto \frac{\bar{x}^{2}}{\ell^{2}}\left(\frac{\ell}{8 \pi r}\right)^{1 / 2} e^{-r / \ell},
$$

where $\ell$ is the correlation length,

$$
\ell=\bar{x} \sqrt{\frac{\tau}{\gamma}}\left[\frac{3}{4}\left(U^{\prime \prime}(\bar{x})+\frac{U^{\prime}(\bar{x})}{\bar{x}}\right)\right]^{1 / 2} .
$$

Thus, a strong potential and/or a large value of $\tau$ increases the value of $\ell$. Also, increasing the average packing fraction (i.e., decreasing the lattice constant $\bar{x}$ ) leads to an increase in $\ell$ through the $U(\bar{x})$ dependence on this quantity. Expression (13) coincides with the result obtained in [46], even if here it is derived under the less restrictive hypothesis of a vibrating (not rigid) lattice. Moreover, at variance with [46], here we are also considering very high average densities with homogeneous (not phase-separated) configurations, allowing us to directly check the scaling of $C(r)$ with $\tau$. On the contrary, when phase separation occurs, the packing fraction $\hat{\phi}$ of the dense regions increases with $\tau$, even at fixed average density $\phi$ (and therefore $\bar{x}$ decreases).

To check predictions (12) and (13), we study the spatial velocity correlation, $C(r)$, for several values of $\tau$ and $\phi$. $C(r)$ for the denser case $(\phi=1.1)$, which corresponds to the solid phase for the whole range of $\tau$ numerically explored, is reported in Fig. 5(c) and reveals a good agreement with 

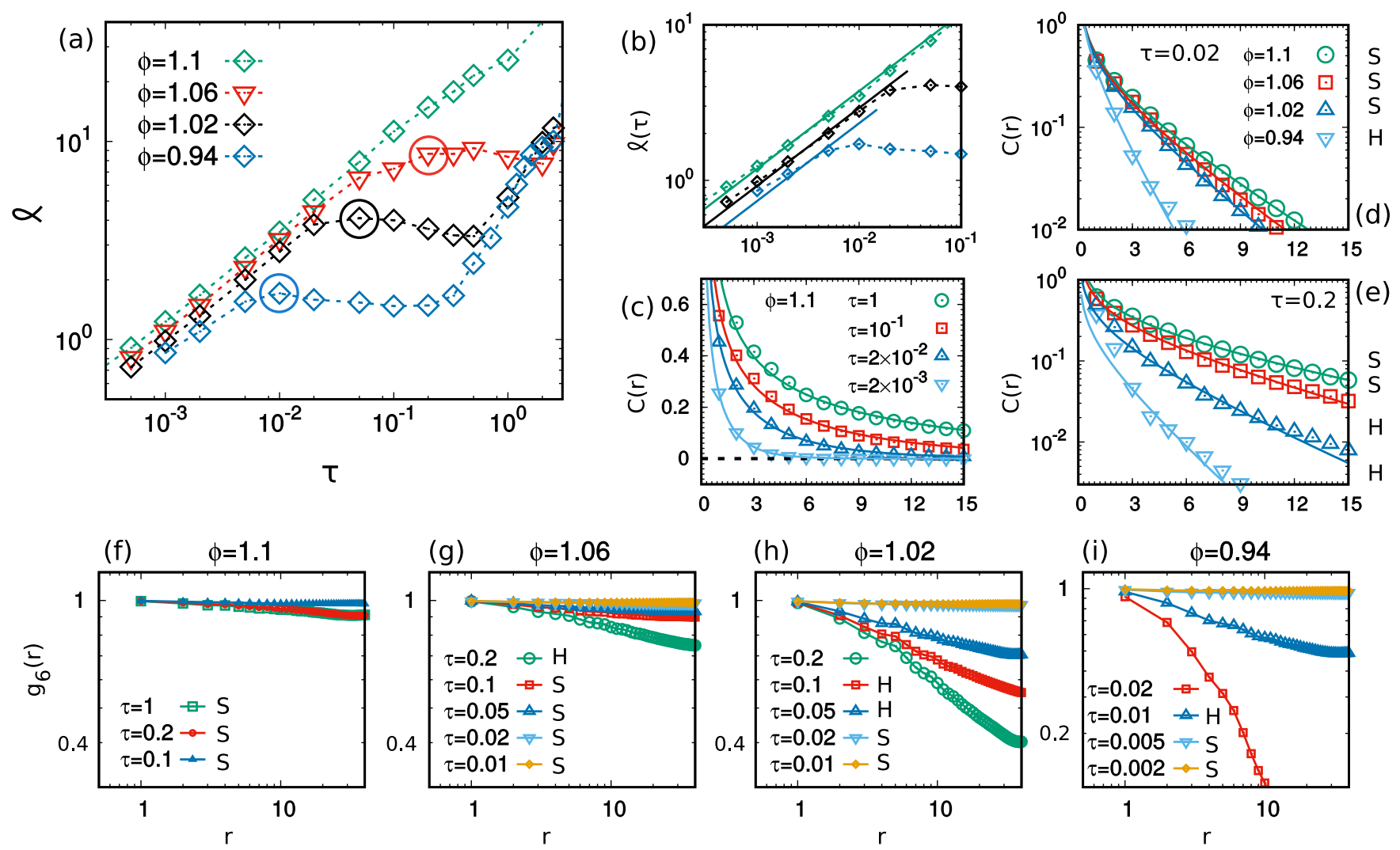

FIG. 5. Spatial correlations of velocity vectors: theory and numerics. (a), (b) Correlation lengths, $\ell$, as a function of $\tau$ for different values of $\phi$ as shown in the legends. The colored circle around a symbol in each curve in (a) is the point at which the solid-hexatic transition is obtained. In particular, (b) zooms into small values of $\tau$ and shows the prediction, (13), as a solid line, fitting the function $A \tau^{1 / 2}, A$ being a fitting parameter. (c)-(e) Plots of the correlation function $C(r)$ for several values of $\phi$ and $\tau$ : for $\phi=1.1$ (i.e., in the solid state) for several values of $\tau$ (c), and for two values of $\tau$ as a function of $\phi[(\mathrm{d})$ and (e), which share the same legend]. In (c)-(e), points were obtained from numerical simulations, and solid lines from the theoretical prediction, Eq. (12). S, solid phase; H, hexatic phase. (f)-(i) $g_{6}(r)$ for different values of $\phi$ and $\tau$. The simulations were realized with $v_{0}=50$.

theory. The correlation length, $\ell$, is reported in Fig. 5(a) (green diamonds) and shows monotonic growth in fairly good agreement with Eq. (13): under these high-density conditions, the defects are not as statistically relevant and do not interfere with the velocity order. The zoom in Fig. 5(b), accompanied by a fit of the numerical data, displays a good agreement with formula (13). A similar analysis reveals discrepancies between theory and simulations when applied to lower values of $\phi$ : the increase in $\ell$ ceases at some value of $\tau$ which depends on $\phi$. We mark with a colored circle the first value of $\tau$ where $\ell$ has reached a plateau and call it $\tau^{*}(\phi)$. Interestingly, this is close (up to numerical errors) to the value of $\tau$ where the solid-hexatic transition takes place (i.e., the fraction of crystalline defects roughly overcomes a given threshold). Here, for completeness, we report the correlation function of $\Psi_{6}$, namely, $g_{6}=\left\langle\Psi_{6}(0) \Psi_{6}^{*}(r)\right\rangle$, in Figs. 5(f) $-5(\mathrm{i})$. This function shows the well-known transition from the solid to the hexatic phases, roughly at $\tau^{*}$, where $g_{6}$ goes from a nearly constant behavior to a power-law decay [71]. For comparison, we also show $C(r)$ as a function of $\phi$ for two values of $\tau$, in Figs. 5(d) and 5(e), where each phase is labeled $\mathrm{S}$ or $\mathrm{H}$ (solid or hexatic) in the legend. In the hexatic phase, $C(r)$ maintains the exponential-like shape predicted in Eq. (12) but decays abruptly much faster in the proximity of the solid-hexatic transition. As a consequence, the agreement between Eq. (13) and the data, shown in Fig. 5(b), holds up to $\tau^{*}$, while for $\tau>\tau^{*}$ the presence of defects invalidates the prediction, (13), since $\ell$ remains nearly constant or decays very slowly. It is also remarkable that a further increase in $\tau$ produces a steep increase in $\ell$. This is likely caused by phase separation and the increase in local density in the clustered regions. Even in this case, we still expect that Eq. (13) holds even if the function $\bar{x}(\tau)$ is unknown.

In conclusion, we have solid arguments to state that a large number of defects occurring in the hexatic or liquid phase are responsible for the saturation of $\ell$. Indeed, in the proximity of a defect, regions with high kinetic energies are present, as shown in the previous section, and as a consequence, the velocities are less correlated. Interestingly, the size $R$ of the orientational domains is not as affected by the lack of orientational order, always revealing monotonic growth with $\tau$ independently of the structural phase.

\section{DISCUSSION AND PERSPECTIVES}

We have studied systems of self-propelled particles at high packing fractions displaying structural properties which resemble the equilibrium liquid, hexatic, and solid phases, 
exploring both small and large persistence regimes. While at small values of the persistence time, $\tau$, many observables behave as at equilibrium, the phenomenology in the highpersistence regime is much richer and displays unexpected dynamical phenomena. The observed dynamical anomalies are included in a new phase diagram upon the introduction of a suitable order parameter encoding the information on the spatial velocity correlations. To the best of our knowledge, this is the first extensive investigation which, going beyond the standard analysis of structural properties, reveals the nonequilibrium peculiarities of packed self-propelled disks.

Specifically, we extend the scenario found in [46] for a moderate packing value to a broad range of packing fractions, confirming that velocities exhibit intriguing patterns and form aligned domains or vortexlike arrangements in all the dense phases of active matter. Remarkably, the lack of translational order hinders the spontaneous alignment in liquid phases where it remains rather low, while the domain size increases considerably in the denser homogeneous phases. This information is included in the phase diagram through a suitable order parameter which quantifies the size of the aligned domains, deduced from the spatial correlations of the velocity orientations. This parameter increases with both the packing fraction, $\phi$, and $\tau$ in the homogeneous phases, while it becomes independent of $\phi$ in the phase-separated regimes. We remark that this scenario is consistent with the absence of alignment phenomenology observed in the homogeneous liquid phase studied in [46] before the occurrence of MIPS, because of the small packing fraction value considered.

We also observe novel dynamical anomalies in the occurrence of large regions whose kinetic energy is highly correlated: these regions become larger when $\tau$ and $\phi$ increase. In addition, high energetic regions form in the proximity of orientational defects of the crystalline aggregates. This scenario is accompanied by a pronounced time intermittency phenomenon, apparently well correlated with the fluctuations of the orientational order parameter. Correlations of the velocity vector are also useful to gain insights into the dynamical features, accounting for both velocity orientation and modulus. Being sensitive to both orientational and translational order, their correlation lengths distinguish between hexatic and solid phases. As an additional analytical result, they can be successfully compared to a mesoscopic theory developed under the assumption of homogeneous density. Deviations from the theory occur when the hexatic phase appears and, with it, a large fraction of defects.

Our observations call for experimental verification. Promising real platforms to confirm such interesting phenomenologies are Janus particles $[15,83]$ and vibrated polar granular disks [17].

In the present work, we have been interested in understanding the dense phase in monodisperse active systems. Binary mixtures are often employed for gaining insight into active glass phases $[62,82,84-90]$. In particular, it has been shown that the spatial velocity correlation function is an input ingredient for developing a self-consistent mode-coupling theory of active matter [86]. Our findings prove that the statistical properties of the velocity field must be taken into account in order to have a complete description of active systems. As a future direction, it would be interesting to understand how the velocity alignment patterns could affect the glassy transition.

The phenomenology of domains with aligned velocities could resemble the scenario of traveling crystals, observed in numerical simulations in Refs. [22,23]. Recently, traveling crystals have been observed in experiments using suspensions of microdisks subjected to vertical vibrations [24]. In such studies, the whole hexagonal pattern moves coherently in space, even though each self-propulsion vector points randomly. Our phenomenology is quite different since far particles (which belong to different domains) move independently, and thus, the whole crystal gets stuck. Instead, the movement of some clusters gives rise to the formation of defects. We find that the whole crystal travels coherently as in Ref. [22] only if the size of the box is smaller than the persistence length of the active motion.

\section{ACKNOWLEDGMENTS}

L.C. thanks M. Cencini and A. Cavagna for fruitful discussions. A.P. and M.P. acknowledge funding from Regione Lazio, Grant Prot. No. 85-2017-15257 (Progetti di Gruppi di Ricerca, Legge 13/2008, art. 4). L.C., U.M.B.M., and A.P. acknowledge support from MIUR PRIN 2017 project 201798CZLJ.

\section{APPENDIX A: VELOCITY DYNAMICS IN THE CASE $T>0$}

The change of variables from $\left(\mathbf{x}_{i}, \mathbf{f}_{i}^{a}\right)$ to $\left(\mathbf{x}_{i}, \mathbf{v}_{i}\right)$, i.e., from Eqs. (1) and (3) to Eqs. (4) and (5), was derived in Ref. [46] in the absence of thermal noise (i.e., for $T=0$ ). The change of variables can be easily generalized to the case $T>0$, as shown in this Appendix. This generalization follows the strategy in Ref. [56], where the result is obtained in the case of the AOUP model for noninteracting particles. Here, the same trick can be adapted to ABP self-propulsion. To overcome the difficulty regarding the time derivation of the thermal noise, we define the variable $\mathbf{v}_{i}=\dot{\mathbf{x}}_{i}-\sqrt{2 T / \gamma} \boldsymbol{w}_{i}$. Taking the derivative with respect to time, the dynamics reads

$$
\begin{gathered}
\dot{\mathbf{x}}_{i}=\mathbf{v}_{i}+\sqrt{2 T / \gamma} \boldsymbol{w}_{i}, \\
\tau \gamma \dot{\mathbf{v}}_{i}=-\gamma \sum_{j=1}^{N} \Gamma_{i j}\left(\mathbf{r}_{i j}\right) \mathbf{v}_{j}+\mathbf{F}_{i}+\tau \gamma \mathbf{k}_{i} \\
-\tau \nabla_{i} \cdot \mathbf{F}_{i} \sqrt{2 T / \gamma} \boldsymbol{w}_{i} .
\end{gathered}
$$

The thermal noise comes into play with two additional noise terms. The first is an additive noise on the dynamics of $\dot{\mathbf{x}}_{i}$, while the second is multiplicative and acts on $\dot{\mathbf{v}}_{i}$. Its space prefactor balances the space-dependent Stokes force in the equilibrium limit $\tau \rightarrow 0$.

\section{APPENDIX B: POSITIONAL, ORIENTATIONAL, AND VELOCITY CORRELATIONS FOR A PERFECT ACTIVE LATTICE}

In order to obtain the correlation functions of the twodimensional system we make two simplifying assumptions in the dynamics, Eq. (1): 
(i) Each component of the active force, $\mathbf{f}_{i}^{a}$, is replaced by independent Ornstein-Uhlenbeck processes, namely, $\boldsymbol{\eta}_{i}$, with equivalent intensity, $v_{0}=\sqrt{D / \tau}$, and persistence time, $1 / D_{r}=\tau$.

(ii) Each particle oscillates around a node of a hexagonal lattice so that the total inter-particle potential can be approximated as the sum of the quadratic terms.

The approximate dynamics reads

$$
\begin{gathered}
\dot{\boldsymbol{\eta}}_{i}(t)=-\frac{1}{\tau} \boldsymbol{\eta}_{i}(t)+\frac{\sqrt{2 D}}{\tau} \xi_{i}(t), \\
\dot{\mathbf{x}}_{i}(t)=\boldsymbol{\eta}_{i}(t)-\sum_{j}^{n . n} \frac{\nabla_{i} U\left(\left|\mathbf{x}_{j}-\mathbf{x}_{i}\right|\right)}{\gamma},
\end{gathered}
$$

where $\nabla_{i} U$ stands for the gradient of the potential $U$ with respect to $\mathbf{x}_{i}$ and the sum involves the nearest neighbors of the lattice node $i$.

Introducing the displacement $\mathbf{u}_{i}$ of particle $i$ with respect to its equilibrium position, $\mathbf{x}_{i}^{0}$, namely,

$$
\mathbf{u}_{i}=\mathbf{x}_{i}-\mathbf{x}_{i}^{0},
$$

we get

$$
\begin{gathered}
\dot{\boldsymbol{\eta}}_{i}(t)=-\frac{1}{\tau} \boldsymbol{\eta}_{i}(t)+\frac{\sqrt{2 D}}{\tau} \boldsymbol{\xi}_{i}(t), \\
\dot{\mathbf{u}}_{i}(t)=\boldsymbol{\eta}_{i}(t)+\frac{K}{\gamma} \sum_{\mathbf{m}}^{n . n}\left(\mathbf{u}_{j}-\mathbf{u}_{i}\right),
\end{gathered}
$$

where $K$ is the strength of the potential in the harmonic approximation, i.e., $U \approx \frac{K}{2}\left(\mathbf{u}_{j}-\mathbf{u}_{i}\right)^{2}$, which reads

$$
2 K=\left(U^{\prime \prime}(\bar{x})+\frac{U^{\prime}(\bar{x})}{\bar{x}}\right) .
$$

In order to solve the problem, we switch to normal coordinates, employing the Fourier-space representation,

$$
\begin{aligned}
& \hat{\mathbf{u}}_{\mathbf{q}}=\frac{1}{N} \sum_{i} \mathbf{u}_{i} e^{-i \mathbf{q} \cdot \mathbf{x}_{i}^{0}}, \\
& \hat{\boldsymbol{\eta}}_{\mathbf{q}}=\frac{1}{N} \sum_{i} \boldsymbol{\eta}_{i} e^{-i \mathbf{q} \cdot \mathbf{x}_{i}^{0}},
\end{aligned}
$$

and obtain

$$
\begin{aligned}
\frac{d}{d t} \hat{\boldsymbol{\eta}}_{\mathbf{q}}(t) & =-\frac{1}{\tau} \hat{\boldsymbol{\eta}}_{\mathbf{q}}+\frac{\sqrt{2 D}}{\tau} \hat{\boldsymbol{\xi}}_{\mathbf{q}}, \\
\frac{d}{d t} \hat{\mathbf{u}}_{\mathbf{q}}(t) & =-\frac{\omega_{\mathbf{q}}^{2}}{\gamma} \hat{\mathbf{u}}_{\mathbf{q}}(t)+\hat{\boldsymbol{\eta}}_{\mathbf{q}},
\end{aligned}
$$

where

$$
\begin{aligned}
\omega_{\mathbf{q}}^{2} & =-2 K\left[\cos \left(q_{x} \bar{x}\right)+2 \cos \left(\frac{1}{2} q_{x} \bar{x}\right) \cos \left(\frac{\sqrt{3}}{2} q_{y} \bar{x}\right)-3\right] \\
& \approx \frac{3}{2} K \bar{x}^{2} q^{2}+O\left(q^{4}\right),
\end{aligned}
$$

with $\mathbf{q}=\left(q_{x}, q_{y}\right)$ vectors of the reciprocal Bravais lattice. Thus, we can easily calculate the steady-state equal-time correlations:

$$
\begin{gathered}
\left\langle\hat{\mathbf{u}}_{\mathbf{q}}(t) \cdot \hat{\mathbf{u}}_{-\mathbf{q}}(t)\right\rangle=\frac{2 D \gamma}{\omega_{\mathbf{q}}^{2}\left(1+\frac{\tau}{\gamma} \omega_{\mathbf{q}}^{2}\right)}, \\
\left\langle\hat{\mathbf{v}}_{\mathbf{q}}(t) \cdot \hat{\mathbf{v}}_{-\mathbf{q}}(t)\right\rangle=\frac{2 D}{\tau} \frac{1}{1+\frac{\tau}{\gamma} \omega_{\mathbf{q}}^{2}}, \\
\left\langle\hat{\mathbf{u}}_{\mathbf{q}}(t) \cdot \hat{\mathbf{v}}_{-\mathbf{q}}(t)\right\rangle=0 .
\end{gathered}
$$

\section{Velocity correlation function}

We now consider the real-space velocity correlation function:

$$
\left\langle\mathbf{v}_{\mathbf{x}} \cdot \mathbf{v}_{\mathbf{x}^{\prime}}\right\rangle=\frac{1}{N^{2}} \frac{2 D}{\tau} \sum_{\mathbf{q}} e^{i \mathbf{q}\left(\mathbf{x}-\mathbf{x}^{\prime}\right)} \frac{1}{\left(1+\frac{\tau}{\gamma} \omega_{\mathbf{q}}^{2}\right)} .
$$

By replacing the lattice sum with a double-dimensional integral and defining $r=\left|\mathbf{x}-\mathbf{x}^{\prime}\right|$, we have

$$
\left\langle\mathbf{v}_{\mathbf{x}} \cdot \mathbf{v}_{\mathbf{x}^{\prime}}\right\rangle \approx \frac{1}{2 \pi} \frac{2 D}{\tau} \frac{\bar{x}^{2}}{\ell^{2}} K_{0}(r / \ell),
$$

where $K_{0}(r / \ell)$ is the zero-order modified Bessel function of the second kind, which has the asymptotic behavior when $r / \ell \gg 1$

$$
K_{0}(r / \ell) \approx\left(\frac{\pi \ell}{2 r}\right)^{1 / 2} e^{-r / \ell}
$$

we find

$$
\left\langle\mathbf{v}_{\mathbf{x}} \cdot \mathbf{v}_{\mathbf{x}^{\prime}}\right\rangle \approx 2 v_{0}^{2} \frac{\bar{x}^{2}}{\ell^{2}}\left(\frac{\ell}{8 \pi r}\right)^{1 / 2} e^{-r / \ell},
$$

where

$$
\ell^{2}=\frac{3 \tau}{2 \gamma} \bar{x}^{2} K=\frac{3 \tau}{4 \gamma} \bar{x}^{2}\left(U^{\prime \prime}(\bar{x})+\frac{U^{\prime}(\bar{x})}{\bar{x}}\right),
$$

which defines the correlation length $\ell$ in the harmonic hexagonal lattice, in agreement with result (13).

\section{Bond angle order and $\psi_{6}$ field in the harmonic crystal}

We define the angle, $\alpha_{\mathbf{x}}$, between the local crystallographic axes and the axes of the ideal lattice [91],

$$
\alpha_{\mathbf{x}}=\frac{1}{2} \nabla \times \mathbf{u}_{\mathbf{x}},
$$

where we have used the continuum representation. In Fourier space we have

$$
\hat{\alpha}_{\mathbf{q}}=\frac{i}{2}\left(q_{x} \hat{u}_{\mathbf{q} y}-q_{y} \hat{u}_{\mathbf{q} x}\right),
$$

while the $\hat{\alpha}_{\mathbf{q}}$ correlation reads

$$
\left\langle\hat{\alpha}_{\mathbf{q}} \hat{\alpha}_{-\mathbf{q}}\right\rangle=\frac{D \gamma}{4} \frac{q^{2}}{\omega_{\mathbf{q}}^{2}} \frac{1}{1+\frac{\tau}{\gamma} \omega_{\mathbf{q}}^{2}} \approx \frac{\sigma^{2}}{6 \bar{x}^{2}} \frac{1}{1+\ell^{2} q^{2}},
$$

where $\sigma^{2}=D \gamma / K$. The real-space $\alpha_{\mathbf{x}}$-correlation function is given by

$$
\begin{aligned}
\left\langle\left(\alpha_{\mathbf{x}}-\alpha_{\mathbf{x}^{\prime}}\right)^{2}\right\rangle & \propto \frac{1}{N^{2}} \sum_{\mathbf{q}} e^{i \mathbf{q}\left(\mathbf{x}-\mathbf{x}^{\prime}\right)} \frac{\sigma^{2}}{6 \bar{x}^{2}} \frac{1}{1+\ell^{2} q^{2}} \\
& \propto\left(\frac{\ell}{8 \pi r}\right)^{1 / 2} \frac{\bar{x}^{2}}{\ell^{2}} e^{-r / \ell},
\end{aligned}
$$


where we have used the expansion for small $\mathbf{q}$ and the asymptotic behavior of $K_{0}(r / l)$. Now, we consider the correlation function of $\psi_{6 \mathbf{x}}=e^{i 6 \alpha_{\mathbf{x}}}$ :

$$
\left\langle\psi_{6 \mathbf{x}} \psi_{6 \mathbf{x}^{\prime}}^{*}\right\rangle=\left\langle e^{i 6 \alpha_{\mathbf{x}}} e^{-i 6 \alpha_{\mathbf{x}^{\prime}}}\right\rangle
$$

Using the form of the $\alpha$ correlation we find

$$
\left\langle\psi_{6 \mathbf{x}} \psi_{6 \mathbf{x}^{\prime}}^{*}\right\rangle=e^{-\frac{1}{2}\left\langle\left(6 \alpha_{\mathbf{x}}-6 \alpha_{\mathbf{x}^{\prime}}\right)^{2}\right\rangle} .
$$

Consequently, the correlator of $\psi_{6}$ does not vanish at $\infty$, i.e., the order is maintained since

$$
\lim _{\left|\mathbf{x}-\mathbf{x}^{\prime}\right| \rightarrow \infty}\left\langle\psi_{6 \mathbf{x}} \psi_{6 \mathbf{x}^{\prime}}^{*}\right\rangle=\text { const. }
$$

This is the expected result since the harmonic lattice always maintains the sixfold coordination number and no disclinations can be created. We note that for this model the velocity correlation and the $\alpha_{\mathbf{x}}$ correlation have the same long-range behavior.
[1] H. Löwen, Phys. Rep. 237, 249 (1994).

[2] U. Gasser, J. Phys.: Condens. Matter 21, 203101 (2009).

[3] N. D. Mermin and H. Wagner, Phys. Rev. Lett. 17, 1133 (1966).

[4] N. D. Mermin, Phys. Rev. 176, 250 (1968).

[5] B. I. Halperin and D. R. Nelson, Phys. Rev. Lett. 41, 121 (1978).

[6] A. P. Young, Phys. Rev. B 19, 1855 (1979).

[7] V. Berezinsky, Sov. Phys. JETP 32, 493 (1971).

[8] J. M. Kosterlitz and D. J. Thouless, J. Phys. C 6, 1181 (1973).

[9] U. Gasser, C. Eisenmann, G. Maret, and P. Keim, ChemPhysChem 11, 963 (2010).

[10] K. Zahn, R. Lenke, and G. Maret, Phys. Rev. Lett. 82, 2721 (1999).

[11] A. L. Thorneywork, J. L. Abbott, D. G. A. L. Aarts, and R. P. A. Dullens, Phys. Rev. Lett. 118, 158001 (2017).

[12] M. C. Marchetti, J. F. Joanny, S. Ramaswamy, T. B. Liverpool, J. Prost, M. Rao, and R. A. Simha, Rev. Mod. Phys. 85, 1143 (2013).

[13] G. Gompper, R. G. Winkler, T. Speck, A. Solon, C. Nardini, F. Peruani, H. Löwen, R. Golestanian, U. B. Kaupp, L. Alvarez et al., J. Phys.: Condens. Matter 32, 193001 (2020).

[14] C. Bechinger, R. Di Leonardo, H. Löwen, C. Reichhardt, G. Volpe, and G. Volpe, Rev. Mod. Phys. 88, 045006 (2016).

[15] N. Klongvessa, F. Ginot, C. Ybert, C. Cottin-Bizonne, and M. Leocmach, Phys. Rev. Lett. 123, 248004 (2019).

[16] J. Deseigne, O. Dauchot, and H. Chaté, Phys. Rev. Lett. 105, 098001 (2010).

[17] G. Briand and O. Dauchot, Phys. Rev. Lett. 117, 098004 (2016).

[18] T. E. Angelini, E. Hannezo, X. Trepat, M. Marquez, J. J. Fredberg, and D. A. Weitz, Proc. Natl. Acad. Sci. USA 108, 4714 (2011).

[19] S. Garcia, E. Hannezo, J. Elgeti, J.-F. Joanny, P. Silberzan, and N. S. Gov, Proc. Natl. Acad. Sci. USA 112, 15314 (2015).

[20] A. Mongera, P. Rowghanian, H. J. Gustafson, E. Shelton, D. A. Kealhofer, E. K. Carn, F. Serwane, A. A. Lucio, J. Giammona, and O. Campàs, Nature 561, 401 (2018).

[21] J. Bialké, T. Speck, and H. Löwen, Phys. Rev. Lett. 108, 168301 (2012).

[22] A. M. Menzel and H. Löwen, Phys. Rev. Lett. 110, 055702 (2013).

[23] A. M. Menzel, T. Ohta, and H. Löwen, Phys. Rev. E 89, 022301 (2014).

[24] G. Briand, M. Schindler, and O. Dauchot, Phys. Rev. Lett. 120, 208001 (2018).

[25] P. Digregorio, D. Levis, A. Suma, L. F. Cugliandolo, G. Gonnella, and I. Pagonabarraga, Phys. Rev. Lett. 121, 098003 (2018).
[26] L. F. Cugliandolo, P. Digregorio, G. Gonnella, and A. Suma, Phys. Rev. Lett. 119, 268002 (2017).

[27] J. Stenhammar, D. Marenduzzo, R. J. Allen, and M. E. Cates, Soft Matter 10, 1489 (2014).

[28] J. U. Klamser, S. C. Kapfer, and W. Krauth, Nat. Commun. 9, 5045 (2018).

[29] G. S. Redner, M. F. Hagan, and A. Baskaran, Phys. Rev. Lett. 110, 055701 (2013).

[30] I. Buttinoni, J. Bialké, F. Kümmel, H. Löwen, C. Bechinger, and T. Speck, Phys. Rev. Lett. 110, 238301 (2013).

[31] J. Palacci, S. Sacanna, A. P. Steinberg, D. J. Pine, and P. M Chaikin, Science 339, 936 (2013).

[32] J. Bialké, T. Speck, and H. Löwen, J. Non-Cryst. Solids 407, 367 (2015).

[33] F. Ginot, I. Theurkauff, F. Detcheverry, C. Ybert, and C. CottinBizonne, Nat. Commun. 9, 696 (2018).

[34] J. T. Siebert, J. Letz, T. Speck, and P. Virnau, Soft Matter 13, 1020 (2017).

[35] T. Speck, Eur. Phys. J.: Spec. Top. 225, 2287 (2016).

[36] P. Chiarantoni, F. Cagnetta, F. Corberi, G. Gonnella, and A. Suma, J. Phys. A, (2020), doi: 10.1088/1751-8121/ab8f3c.

[37] Y. Fily and M. C. Marchetti, Phys. Rev. Lett. 108, 235702 (2012).

[38] M. E. Cates and J. Tailleur, Annu. Rev. Condens. Matter Phys. 6, 219 (2015).

[39] G. Gonnella, D. Marenduzzo, A. Suma, and A. Tiribocchi, C.R. Phys. 16, 316 (2015).

[40] Z. Ma, M. Yang, and R. Ni, arXiv:2004.02376.

[41] A. P. Solon, Y. Fily, A. Baskaran, M. E. Cates, Y. Kafri, M. Kardar, and J. Tailleur, Nat. Phys. 11, 673 (2015).

[42] A. P. Solon, J. Stenhammar, R. Wittkowski, M. Kardar, Y. Kafri, M. E. Cates, and J. Tailleur, Phys. Rev. Lett. 114, 198301 (2015).

[43] J. Bialké, J. T. Siebert, H. Löwen, and T. Speck, Phys. Rev. Lett. 115, 098301 (2015).

[44] A. Patch, D. M. Sussman, D. Yllanes, and M. C. Marchetti, Soft Matter 14, 7435 (2018).

[45] S. Mandal, B. Liebchen, and H. Löwen, Phys. Rev. Lett. 123, 228001 (2019).

[46] L. Caprini, U. Marini Bettolo Marconi, and A. Puglisi, Phys. Rev. Lett. 124, 078001 (2020).

[47] A. Puglisi, A. Gnoli, G. Gradenigo, A. Sarracino, and D. Villamaina, J. Chem. Phys. 136, 014704 (2012).

[48] A. Manacorda, Lattice Models for Fluctuating Hydrodynamics in Granular and Active Matter (Springer-Verlag, Berlin, 2018).

[49] A. Plati, A. Baldassarri, A. Gnoli, G. Gradenigo, and A. Puglisi, Phys. Rev. Lett. 123, 038002 (2019). 
[50] T. F. F. Farage, P. Krinninger, and J. M. Brader, Phys. Rev. E 91, 042310 (2015).

[51] T. Vicsek and A. Zafeiris, Phys. Rep. 517, 71 (2012).

[52] G. Grégoire and H. Chaté, Phys. Rev. Lett. 92, 025702 (2004).

[53] A. Martín-Gómez, D. Levis, A. Daz-Guilera, and I. Pagonabarraga, Soft Matter 14, 2610 (2018).

[54] K.-D. N. T. Lam, M. Schindler, and O. Dauchot, New J. Phys. 17, 113056 (2015).

[55] F. Giavazzi, M. Paoluzzi, M. Macchi, D. Bi, G. Scita, M. L. Manning, R. Cerbino, and M. C. Marchetti, Soft Matter 14, 3471 (2018).

[56] L. Caprini and U. M. B. Marconi, Soft Matter 14, 9044 (2018).

[57] U. M. B. Marconi and C. Maggi, Soft Matter 11, 8768 (2015).

[58] U. M. B. Marconi, A. Puglisi, and C. Maggi, Sci. Rep. 7, 46496 (2017).

[59] U. M. B. Marconi, N. Gnan, M. Paoluzzi, C. Maggi, and R. Di Leonardo, Sci. Rep. 6, 23297 (2016).

[60] É. Fodor, C. Nardini, M. E. Cates, J. Tailleur, P. Visco, and F. van Wijland, Phys. Rev. Lett. 117, 038103 (2016).

[61] R. Wittmann, F. Smallenburg, and J. M. Brader, J. Chem. Phys. 150, 174908 (2019).

[62] L. Berthier, E. Flenner, and G. Szamel, J. Chem. Phys. 150, 200901 (2019).

[63] L. Caprini, U. M. B. Marconi, A. Puglisi, and A. Vulpiani, J. Stat. Mech.: Theory Exp. (2019) 053203.

[64] E. Woillez, Y. Kafri, and V. Lecomte, arXiv:1912.04010.

[65] L. Caprini and U. M. B. Marconi, Soft Matter 15, 2627 (2019).

[66] C. Maggi, M. Paoluzzi, L. Angelani, and R. Di Leonardo, Sci. Rep. 7, 17588 (2017).

[67] L. Caprini, E. Hernández-García, C. López, and U. M. B. Marconi, Sci. Rep. 9, 1 (2019).

[68] S. Das, G. Gompper, and R. G. Winkler, New J. Phys. 20, 015001 (2018).

[69] L. Caprini, U. M. B. Marconi, and A. Puglisi, Sci. Rep. 9, 1386 (2019).

[70] L. Caprini, F. Cecconi, and U. Marini Bettolo Marconi, J. Chem. Phys. 150, 144903 (2019).
[71] L. F. Cugliandolo and G. Gonnella, arXiv:1810.11833.

[72] H. Chaté, F. Ginelli, G. Grégoire, and F. Raynaud, Phys. Rev. E 77, 046113 (2008).

[73] S. Mishra, A. Baskaran, and M. C. Marchetti, Phys. Rev. E 81, 061916 (2010).

[74] A. M. Menzel, Phys. Rev. E 85, 021912 (2012).

[75] T. Vicsek, A. Czirók, E. Ben-Jacob, I. Cohen, and O. Shochet, Phys. Rev. Lett. 75, 1226 (1995).

[76] A. Cavagna and I. Giardina, Annu. Rev. Condens. Matter Phys. 5, 183 (2014).

[77] F. Peruani, A. Deutsch, and M. Bär, Phys. Rev. E 74, 030904(R) (2006).

[78] F. Ginelli, F. Peruani, M. Bär, and H. Chaté, Phys. Rev. Lett. 104, 184502 (2010).

[79] M. Abkenar, K. Marx, T. Auth, and G. Gompper, Phys. Rev. E 88, 062314 (2013).

[80] F. Peruani, J. Phys. Soc. Jpn. 86, 101010 (2017).

[81] T. Bohr, M. H. Jensen, G. Paladin, and A. Vulpiani, Dynamical Systems Approach to Turbulence (Cambridge University Press, Cambridge, UK, 2005).

[82] R. Mandal, P. J. Bhuyan, P. Chaudhuri, C. Dasgupta, and M. Rao, Nat. Commun. 11, 2581 (2020).

[83] S. C. Takatori, R. De Dier, J. Vermant, and J. F. Brady, Nat. Commun. 7, 10694 (2016).

[84] L. Berthier and G. Biroli, Rev. Mod. Phys. 83, 587 (2011).

[85] L. M. C. Janssen, J. Phys. Condens. Matter 31, 503002 (2019).

[86] G. Szamel, E. Flenner, and L. Berthier, Phys. Rev. E 91, 062304 (2015).

[87] L. Berthier, E. Flenner, and G. Szamel, New J. Phys. 19, 125006 (2017).

[88] R. Ni, M. A. C. Stuart, and M. Dijkstra, Nat. Commun. 4, 2704 (2013).

[89] R. Mandal, P. J. Bhuyan, M. Rao, and C. Dasgupta, Soft Matter 12, 6268 (2016).

[90] S. K. Nandi, R. Mandal, P. J. Bhuyan, C. Dasgupta, M. Rao, and N. S. Gov, Proc. Natl. Acad. Sci. USA 115, 7688 (2018).

[91] J. D. Brock, Bond-Orientational Order in Condensed Matter Systems (Springer-Verlag, Berlin, 1992), pp. 1-31. 